Revista Colombiana de Obstetricia y Ginecología Vol. 62 No. 4 • Octubre-Diciembre 2011 •(331-337)

Reporte de caso

\title{
COMPLEJO LIMB-BODY WALL EN EL HOSPITAL UNIVERSITARIO SAN IGNACIO EN BOGOTÁ (COLOMBIA). REPORTE DE CASO Y REVISIÓN DE LA LITERATURA
}

\section{Limb-body wall complex in the San Ignacio Teaching Hospital at Bogota, Colombia: a case report and literature review}

Mercedes Olaya, M.D.*, Ana Milena Gómez, M.D.**

Recibido: marzo 30/11 - Aceptado: noviembre 17/11

\section{RESUMEN}

Introducción: las alteraciones del cordón umbilical están asociadas con diversas complicaciones de la gestación a corto y a largo plazo, algunas con pocas probabilidades de sobrevida y relacionadas con lesiones fetales múltiples. El complejo Limb-Body Wall (LBWC), compuesto por defectos de la pared corporal lateral y anomalías por acortamiento de miembros, es una de tales complicaciones, cuyas aún discutidas etiologías, pobre pronóstico y posibilidad de recurrencia hacen que cobre importancia en el diagnóstico ecográfico, la atención neonatal y el abordaje de la autopsia.

Objetivos: reportar un caso de LBWC y revisar la literatura publicada sobre su etiología, características clínicas y diagnóstico, en el contexto de las complicaciones asociadas con las alteraciones del cordón umbilical.

Materiales y métodos: se presenta el caso clínico de una recién nacida de primer embarazo de madre adolescente quien consultó a una entidad de nivel

\footnotetext{
* Médica Cirujana Especialista en Patología. Pasantía en Patología Pediátrica. Estudiante de Doctorado en Ciencias Biológicas. Profesora Asociada. Departamento de Patología, Facultad de Medicina, Pontificia Universidad Javeriana. Bogotá (Colombia). Correo electrónico: molayac@hotmail.com

** Médica cirujana. Residente de segundo año de Genética Médica. Facultad de Medicina, Pontificia Universidad Javeriana. Bogotá (Colombia).
}

referencia y alta complejidad, recién nacida que falleció a los pocos minutos de nacer; presentaba defecto de la pared abdominal, escoliosis y esbozo de miembro superior izquierdo, asociado con cordón umbilical de $6 \mathrm{~cm}$.

Se realizó una revisión de la literatura publicada en las bases de datos Medline vía PubMed, Ovid e Hinari.

Conclusión: el LBWC se asocia con un índice importante de morbimortalidad y con la expresión de un cordón excesivamente corto; por tanto, cobra gran relevancia hacer un diagnóstico oportuno y brindar asesoría genética adecuada a las familias.

Palabras clave: cordón umbilical, anomalías de la pared abdominal, ultrasonografía prenatal, asesoramiento genético.

\section{SUMMARY}

Introduction: umbilical cord alterations are associated with several short and long-term complications during pregnancy, some involving little chance of survival related to multiple foetal lesions. One of those anomalies is the Limb-Body Wall Complex (LBWC) which consists of lateral body wall defects and anomalies due to shortening of the limbs. Its still discussed aetiology, poor prognosis and possible recurrence make it important in 
ecographic diagnosis, neonatal attention and the approach during autopsy.

Objectives: reporting a case of LBWC and reviewing the literature published about its aetiology, clinical characteristics and diagnosis within the context of the complications associated with umbilical cord alterations.

Materials and methods: a clinical case of a female newborn resulting from an adolescent mother's first pregnancy who consulted a reference and high complexity entity is presented. The newborn died a few minutes after being born, presenting a defect of the abdominal wall, scoliosis and upper left limb stump associated with a $6 \mathrm{~cm}$ umbilical cord.

The literature published in Medline data bases was reviewed using PubMed, Ovid and Hinari.

Conclusion: LBWC is an expression of an excessively short cord and is associated with important morbidity-mortality. Thus an early diagnosis must be made and suitable genetic advice given to families.

Key words: umbilical cord, abdominal wall anomaly, prenatal ultrasonography, genetic consultation.

\section{INTRODUCCIÓN}

Las condiciones de la vida fetal intrauterina determinan, además del desarrollo y sobrevivencia fetal, la adecuada adaptación del recién nacido y la aparición de diversas e importantes enfermedades de la adultez. ${ }^{1,2}$ Un inadecuado desarrollo fetal depende del genoma y de la epigenética. ${ }^{3}$ El cordón umbilical, conexión entre la madre y el hijo, juega un importante papel en estas relaciones. ${ }^{4}$ La longitud del cordón umbilical y su enrollamiento tradicionalmente son índices de la movilidad fetal: ${ }^{5,6}$ tanto los cordones cortos como los largos tienen complicaciones obstétricas y la longitud anormal del cordón umbilical es considerada un factor principal que predispone a complicaciones periparto. ${ }^{7}$ Los cordones muy cortos se relacionan con anormalidades fetales, particularmente con defectos de la pared abdominal, deformidades de las extremidades y de la columna vertebral. Además de las malformaciones y de las complicaciones neonatales, se ha asociado con pobre coeficiente intelectual y otras complicaciones neurológicas. ${ }^{8}$ El complejo Limb-Body Wall constituye una de las expresiones más graves de fetos afectados por un cordón excesivamente corto. Esta entidad se caracteriza por defectos de la pared corporal lateral asociados con anomalías por acortamiento de miembros y con alta morbimortalidad de los fetos afectados, desencadenándose la mayoría de los casos en abortos o mortinatos. Debido a la alta mortalidad asociada y al poco conocimiento por parte del cuerpo médico, podría decirse que la frecuencia de esta enfermedad se encuentra subestimada, por lo cual cobra importancia hacer una revisión exhaustiva de la literatura publicada para brindar herramientas adecuadas a los médicos que tratan este tipo de patologías (ginecólogos, genetistas, patólogos, neonatólogos, entre otros) para realizar un adecuado abordaje y manejo. Se informa de un caso estudiado en nuestro servicio de patología, que presentaba los criterios diagnósticos compatibles con esta enfermedad, y se revisan las alteraciones relacionadas con cordones muy cortos, en este caso el LBWC, con el objetivo de revisar la literatura respecto a su epidemiologia, características clínicas, etiología y diagnóstico.

\section{REPORTE DE CASO}

Recién nacido recibido en el servicio de patología del Hospital Universitario San Ignacio, entidad privada de nivel IV de complejidad que atiende pacientes de los regímenes contributivo y subsidiado, de todos los niveles socioeconómicos. Producto del primer embarazo de madre de 17 años con ecografía de 34 semanas en la que se observó feto con defecto de pared abdominal anterior y pie equino varo bilateral; se realizó cesárea programada a las 37 semanas de gestación; el recién nacido falleció a los pocos minutos 
de vida. En el estudio radiológico se observó escoliosis dorsolumbar, ausencia de extremidad superior izquierda y gran masa que involucra hemitórax, hipocondrio y flanco izquierdo. En la necropsia se determinó que el recién nacido era de sexo femenino, con defecto de la pared toracoabdominal izquierda con exposición de órganos: hígado, bazo, estómago, intestino grueso, intestino delgado, páncreas, pulmón izquierdo, corazón, vejiga, ovario y útero, los cuales estaban parcialmente contenidos en la bolsa amniótica. El miembro superior izquierdo era un esbozo de $1 \mathrm{~cm}$. Se evidenció cordón umbilical corto $(6 \mathrm{~cm})$, y pulmones con lobulación anómala (figuras 1-3).

Figura 1. Recién nacido unido a la placenta. Escoliosis dorsolumbar severa.

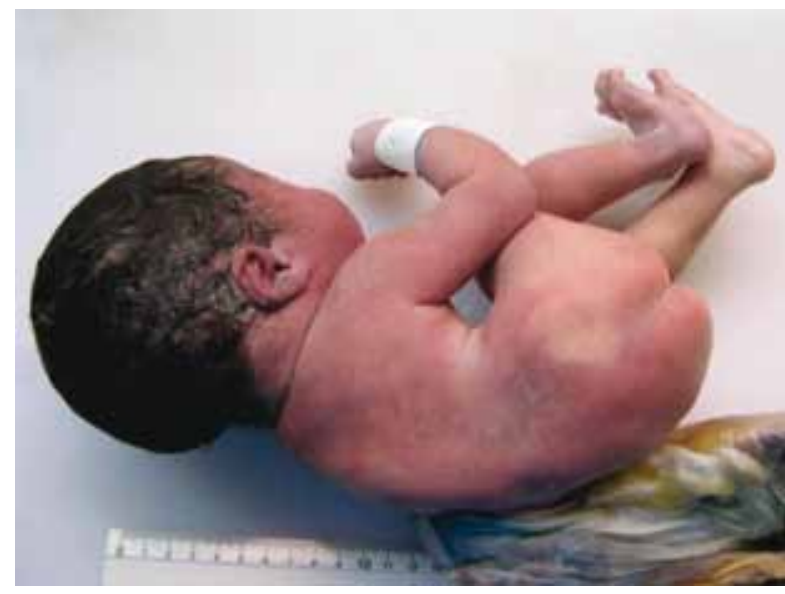

Figura 2. Cordón umbilical de $6 \mathrm{~cm}$.

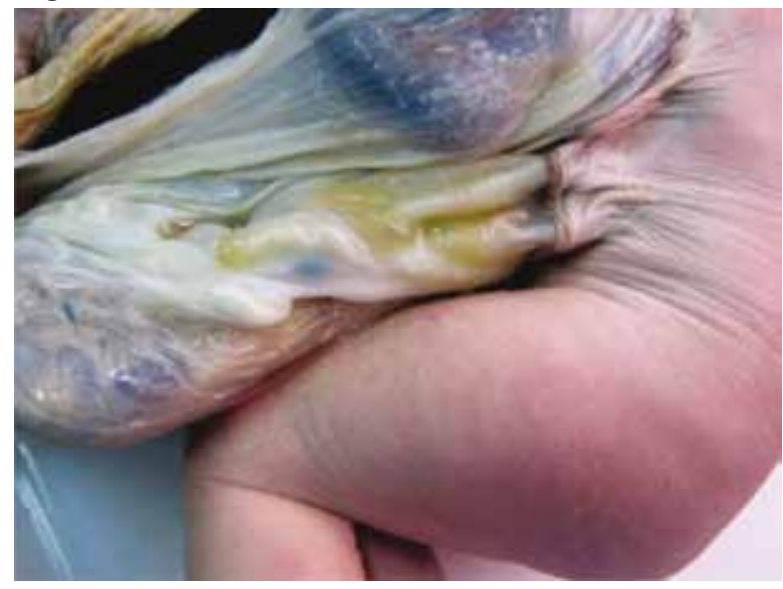

Figura 3. Esbozo de miembro superior izquierdo y bolsa amniótica.

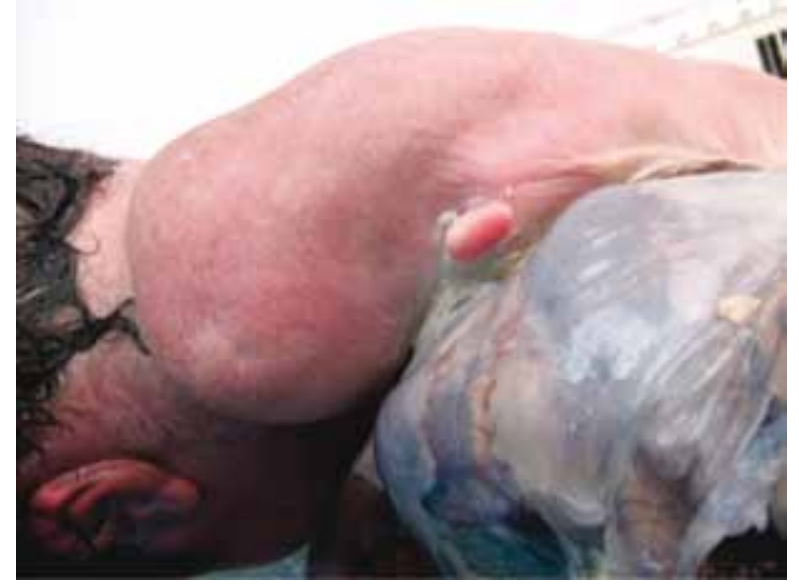

La necropsia, la toma de fotografías y otros estudios complementarios se realizaron con el consentimiento informado de los padres. Las fotografías protegen la identidad del recién nacido y su familia.

\section{MATERIALES Y MÉTODOS}

Se realizó una revisión de la literatura publicada en las bases de datos Medline vía PubMed, Ovid e Hinari, utilizando las palabras clave: "limb-body wall complex, umbilical cord abnormalities, abdominal wall abnormalities, prenatal ultrasonography". Se evaluaron artículos en inglés y en español sin restricción de fecha.

\section{RESULTADOS}

Se obtuvieron aproximadamente 65 referencias y se incluyeron las referencias de artículos de revisión, reportes de casos, hallazgos de patología y diagnóstico temprano.

\section{DISCUSIÓN}

El LBWC consiste en un grupo heterogéneo de malformaciones fetales que incluyen defectos de la pared corporal lateral (abdominosquisis o toracosquisis) y anomalías por acortamiento de miembros. ${ }^{9}$ La mayoría de los casos son abortos o mortinatos. Se ha estimado una prevalencia de $1 / 10000$ a $1 / 40000$ nacimientos, ${ }^{10}$ aunque puede que estos cálculos no reflejen lo que realmente 
sucede, dada la alta mortalidad temprana en el embarazo. Esta patología se distribuye igualmente en ambos sexos. ${ }^{11}$ Se define como "complejo", ya que hay alteración en "campos del desarrollo" del embrión, ${ }^{12}$ los cuales han sido definidos por Opitz ${ }^{13}$ como partes del embrión en las que el desarrollo es controlado y coordinado con un orden espacial, sincronizado temporalmente y de manera jerárquica. Los criterios diagnósticos descritos por Van Allen y colaboradores en $1987,{ }^{14}$ incluyen: 1) exencefalia/ encefalocele y hendiduras faciales, 2) toraco y/o abdominosquisis y 3) defectos en miembros. Se requieren dos de los tres criterios y debe presentarse el defecto en la pared que demuestre la ausencia de una porción de la pared corporal, incluyendo piel, músculo y peritoneo ${ }^{15}$; ocurre en varias formas, desde onfalocele y gastrosquisis hasta malformaciones más complejas. ${ }^{16-20}$

Pueden también asociarse malformaciones internas en el 95\% de los casos, por ejemplo, anomalías urogenitales, ano imperforado y cifoescoliosis,$^{21}$ esta última atribuida a aplasia o hipoplasia de los músculos paravertebrales. ${ }^{21}$ Otros defectos pueden ser malformaciones en el sistema nervioso central (SNC), hendiduras faciales, malformaciones cardiovasculares y ausencia o hipoplasia del diafragma. ${ }^{15}$ Los defectos de los miembros están presentes en el 96\% de los casos, ${ }^{21}$ siendo más comunes los defectos en miembros inferiores, que pueden ser pie equino, surcos anulares, oligodactilia, rotación anormal, sirenomelia y, eventualmente, ausencia completa de una pierna o de una hemipelvis. Además, hay anormalidades como cordón umbilical corto o cordón bivascular ${ }^{15}$ hasta en el 90\% de los casos, dado que la formación de la pared corporal normal se encuentra fuertemente asociada con la formación del cordón umbilical. ${ }^{22-24}$

Gilbert-Barness y colegas dividieron los casos de cordón corto en grupos etiológicos: ${ }^{25}$ grupo I: cordón corto con bandas amnióticas, secuencia ADAM $;^{26,27}$ grupo II: cordón corto menor de 10 $\mathrm{cm}$, con defecto de la pared abdominal, cuerpo fetal "inclinado" llamado pleurosomo-schisi (apertura lateral del tronco $)^{28}$ y ausencia de extremidad; grupo III: cordón corto con severo defecto de cierre de la línea media, con onfalocele, ectopia cordis, extrofia vesical, labio y/o paladar hendido y defectos del tubo neural; grupo IV: cordón corto en gestación gemelar anormal; grupo V: cordón corto con desorden neuromuscular, artrogriposis y defectos de las extremidades; grupo VI: cordón corto con defecto de la pared abdominal y onfalocele. Con base en estos grupos etiológicos puede decirse que el caso de LBWC reportado hace parte del grupo II. El cordón umbilical corto es considerado como "frecuente" por Jones y colaboradores en la secuencia ADAM, LBWC, síndrome NeuLaxova, fenotipo Pena-Shokeir y en la dermopatía restrictiva; se considera "ocasional” en la secuencia disruptiva amioplasia congénita y el síndrome de pterigio múltiple letal. ${ }^{29}$

Se han descrito dos fenotipos principales en la literatura: ${ }^{9,30,31}$ 1) fetos con defectos craneofaciales: muestran dos características específicas: a) encefalocele o exencefalia, siempre asociada con hendiduras faciales, b) bandas amnióticas y/o adhesiones amnióticas anchas entre el defecto craneano y la placenta; 2) fetos sin defectos craneofaciales: usualmente presentan anomalías urogenitales, atresia anal, meningocele lumbosacro y anomalías placentarias con cordón umbilical corto, persistencia de celoma extraembrionario y amnios intacto.

La etiología del LBWC es desconocida, pero se han propuesto diferentes teorías: 1) defectos en el disco germinal que conducen a un plegamiento embrionario anormal, esto lleva a un cierre defectuoso de la pared abdominal, desarrollo anormal del cordón umbilical y persistencia de celoma extraembrionario que se comunica con la cavidad abdominal; 2) ruptura temprana del amnios y formación de bandas amnióticas, con posible compresión mecánica entre las semanas $3^{\mathrm{a}}$ y $5^{\mathrm{a}}$ de vida intrauterina; ${ }^{21}$ 3) disrupción vascular, que explicaría la alta incidencia de malformaciones 
internas, tal vez secundaria a hipoplasia de vasos sanguíneos (por disgenesia embrionaria), más que ser el factor etiológico primario..$^{10}$ Otros han agregado como etiopatogenia, un disco germinal defectuoso con alteraciones generalizadas en el desarrollo embrionario temprano. ${ }^{11}$ Se han hecho estudios experimentales en busca de las señales del cierre de la pared abdominal, en los que se atribuye un papel importante al factor de transcripción AP2-alfa. ${ }^{32}$

No hay teratogénicos conocidos ni anomalías genéticas ${ }^{11,32}$ o cromosómicas relacionadas y el riesgo de recurrencia para un embarazo posterior es muy bajo,${ }^{30}$ sin embargo, se ha descrito recurrencia familiar de un hermano con LBWC y el otro con secuencia de bandas amnióticas, ${ }^{33}$ y otro caso de dos hermanos con LBWC. ${ }^{11,34}$ Se han observado algunas asociaciones con el uso de anticonceptivos orales durante los primeros meses de embarazo ${ }^{11}$ y consumo de cocaína. ${ }^{21,35}$ Además, también se ha reportado LBWC posterior a fertilización in vitro en gestación única ${ }^{36}$ y gemelar. ${ }^{37}$

Dado el pobre pronóstico de esta enfermedad, en comparación con entidades que también implican defectos en la pared abdominal, es importante establecer un correcto diagnóstico diferencial. ${ }^{38}$ Se requiere un cuidadoso estudio ultrasonográfico del feto cuando se sospeche un defecto ventral de la pared abdominal, 9,15,16,21,30,39,40 siendo la mayoría de las características fácilmente reconocibles; solamente el oligohidramnios severo o una estructura complicada de las vísceras herniadas impiden una adecuada visualización de las márgenes fetales. ${ }^{15}$ Se observa una masa anterior o lateral al abdomen y/o tórax del feto, usualmente representada por el hígado y los intestinos. ${ }^{41}$ El hallazgo de bandas amnióticas y una conexión anormal entre el feto y la placa coriónica pueden ser interpretados como un signo de ruptura de la membrana amniótica y persistencia de celoma extraembrionario. Un punto importante para distinguir entre los diferentes defectos de la pared abdominal anterior es la posición del defecto en relación con la inserción del cordón umbilical: en LBWC se presenta un gran defecto lateral excéntrico, mientras que en la gastrosquisis se suele observar un defecto paraumbilical derecho, y el onfalocele y la pentalogía de Cantrell comprometen la línea media en el sitio de inserción del cordón. ${ }^{42}$ Se suele identificar cifoescoliosis severa y alteraciones del cordón en la mayoría de los casos. ${ }^{23,24}$ La detección de un amplio rango de malformaciones en miembros completa el cuadro de LBWC y cuando hay presencia de defectos craneanos o hendiduras faciales se logran reconocer por ecografía. Sin embargo, la extensión de los defectos internos es difícil de demostrar; ${ }^{42,43}$ con resonancia nuclear magnética se pueden determinar con más exactitud las masas pélvicas y otras anomalías. ${ }^{44}$ Se puede identificar el fenotipo según el conjunto de características observadas en la ecografía. ${ }^{45}$ También se han estudiado otras aproximaciones paraclínicas y se ha encontrado que las concentraciones antenatales elevadas de alfafetoproteína sérica pueden sugerir el diagnóstico. ${ }^{40}$ Por último y para completar la aproximación diagnóstica a esta entidad, es crucial el examen de la placenta y del cordón umbilical. ${ }^{46}$

\section{CONCLUSIÓN}

Se hace el informe de un caso con características clínicas de LBWC, en el cual se pudo hacer un adecuado diagnóstico clínico y por imágenes, de acuerdo con lo reportado en la literatura; este caso y la revisión realizada ilustran la importancia de reconocer este tipo de patologías para hacer un oportuno y adecuado diagnóstico y una correcta asesoría a las familias.

\section{REFERENCIAS}

1. Godfrey KM, Barker DJ. Fetal programming and adult health. Public Health Nutr 2001;4:611-24.

2. Nilsson PM, Holmäng A. Developmental origins of adult disease: an introduction. J Intern Med 2007;261:410-1.

3. Lahti J, Räikkönen K, Heinonen K, Pesonen A-K, Kajantie E, Forsén T, et al. Body size at birth and socio-economic status in childhood: implications for 
Cloninger's psychobiological model of temperament at age 60. Psychiatry Res 2008;160:167-74.

4. Sankaran S, Kyle PM. Aetiology and pathogenesis of IUGR. Best Pract Res Clin Obstet Gynaecol 2009;23:765-77.

5. Moessinger AC, Blanc WA, Marone PA, Polsen DC. Umbilical cord length as an index of fetal activity: experimental study and clinical implications. Pediatr Res 1982;16:109-12.

6. Bolisetty S, Koh TH, Hammond S, Panaretto K, Whitehall J. Correlation of umbilical cord weight with birth weight. Arch Dis Child Fetal Neonatal Ed 2002;86:F140.

7. Stefos T, Sotiriadis A, Vasilios D, Tsirkas P, Korkontzelos I, Avgoustatos F, et al. Umbilical cord length and parity--the Greek experience. Eur J Obstet Gynecol Reprod Biol 2003;107:41-4.

8. Scher MS. Fetal and neonatal neurologic consultations: identifying brain disorders in the context of fetalmaternal-placental disease. Semin Pediatr Neurol 2001;8:55-73.

9. Caksen H, Atas B, Tuncer O, Odabas D, Dal H. A case of limb-body wall complex diagnosed in utero. J Emerg Med 2003;25:104-6.

10. Sahinoglu Z, Uludogan M, Arik H, Aydin A, Kucukbas M, Bilgic R, et al. Prenatal ultrasonographical features of limb body wall complex: a review of etiopathogenesis and a new classification. Fetal Pediatr Pathol 2007;26:135-51.

11. Plakkal N, John J, Jacob SE, Chithira J, Sampath S. Limb body wall complex in a still born fetus: a case report. Cases J 2008;1:86.

12. Tanteles GA, Suri M. Classification and aetiology of birth defects. Paediatrics and Child Health 2007; 17:233-43.

13. Opitz JM. The developmental field concept in clinical genetics. J Pediatr 1982;101:805-9.

14. Van Allen MI, Curry C, Gallagher L. Limb body wall complex: I. Pathogenesis. Am J Med Genet 1987;28:529-48.

15. Pumberger W, Schaller A, Bernaschek G. Limbbody wall complex: a compound anomaly pattern in body-wall defects. Pediatr Surg Int 2001;17:48690.

16. Aspelund G, Langer JC. Abdominal wall defects. Current Paediatrics 2006;16:192-8.

17. Bawa M, Samalad VM, Kanojia RP, Rao KL. Partial umbilical exstrophy with cecal patch and intact hindgut entering a cloaca: a new variant. J Pediatr Surg 2011;46:244-6.

18. Chen CP. Syndromes and disorders associated with omphalocele (II): OEIS complex and Pentalogy of Cantrell. Taiwan J Obstet Gynecol 2007;46:103-10.

19. Dillon E, Renwick M. The antenatal diagnosis and management of abdominal wall defects: the northern region experience. Clin Radiol 1995;50:855-9.

20. Kalousek DK. Embryonic and fetal wastage. En: Stocker JT, Dehner LP, editors. Pediatric Pathology. 2nd. ed. Philadelphia: Lippincott Williams and Wilkins; 2001. p. 55-80.

21. Managoli S, Chaturvedi P, Vilhekar KY, Gagane N. Limb body wall complex. Indian Pediatr 2003;40:891-4.

22. Arici DS, Perçin F, Ozer H, Aslan M, Cetin A. Limb body wall defect associated with rare cardiac anomalies. Pediatr Int 2004;46:85-7.

23. Negishi H, Yaegashi M, Kato EH, Yamada H, Okuyama K, Fujimoto S. Prenatal diagnosis of limb-body wall complex. J Reprod Med 1998;43:659-64.

24. Patten RM, Van Allen M, Mack LA, Wilson D, Nyberg D, Hirsch J, et al. Limb-body wall complex: in utero sonographic diagnosis of a complicated fetal malformation. AJR Am J Roentgenol 1986;146:1019-24.

25. Frias J, Gilbert-Barness E. Disruptions. En: GilbertBarness E, editor. Potter's Pathology of the Fetus, Infant and Child. Second ed. Philadelphia: Elsevier; 2007.

26. Gordillo G, Olaya M, Zarante I. Bandas amnióticas y el proceso disruptivo-mecánico producido durante el periodo fetal. Latin American Journal of Dysmorphology 2008;1:23-5.

27. Halder A. Amniotic band syndrome and/or limb body wall complex: split or lump. The Application of Clinical Genetics 2010;3:7-15.

28. Brantberg A. Fetal and perinatal implications of anomalies in the gastrointestinal tract and the abdominal wall. Thesis for the degree of doctor medicinae. Trondheim, Norway: Norwegian University of Science and Technology; 2007.

29. Jones KL. Smith's recognizable patterns of human malformation. 6th ed. Philadelphia: Elsevier; 2006.

30. Prasun P, Behera BK, Pradhan M. Limb body wall complex. Indian J Pathol Microbiol 2008;51:255-6.

31. Russo R, D’Armiento M, Angrisani P, Vecchione R. Limb body wall complex: a critical review and a nosological proposal. Am J Med Genet 1993;47:893-900. 
32. Brewer S, Williams T. Loss of AP-2alpha impacts multiple aspects of ventral body wall development and closure. Dev Biol 2004;267:399-417.

33. Levy R, Lacombe D, Rougier Y, Camus E. Limb body wall complex and amniotic band sequence in sibs. Am J Med Genet A 2007;143A:2682-7.

34. Luehr B, Lipsett J, Quinlivan JA. Limb-body wall complex: a case series. J Matern Fetal Neonatal Med 2002;12:132-7.

35. Viscarello RR, Ferguson DD, Nores J, Hobbins JC. Limb-body wall complex associated with cocaine abuse: further evidence of cocaine's teratogenicity. Obstet Gynecol 1992;80:523-6.

36. Chen CP, Tsai FJ, Chen CY, Lin HH, Wang W. Limb-body wall complex with craniofacial defects after ovarian stimulation. Taiwan J Obstet Gynecol 2008;47:474-5.

37. Chen CP, Lee MS, Tsai FJ, Huang MC, Chern SR, Wang W. Limb-body wall complex in one fetus of a dizygotic twin pregnancy conceived by egg donation, in vitro fertilization and embryo transfer: prenatal diagnosis and literature review. Taiwan J Obstet Gynecol 2009;48:446-50.

38. Duddaiwar VA, Geeta V. Limb body wall complex: antenatal sonographic diagnosis. Eur Ultrasound 1997;5:3.
39. Kamata S, Ishikawa S, Usui N, Kitayama Y, Sawai T, Okuyama H, et al. Prenatal diagnosis of abdominal wall defects and their prognosis. J Pediatr Surg 1996;31:267-71.

40. Roberts N, Bhide A. Ultrasound prenatal diagnosis of structural abnormalities. Obstet Gynaecol Reprod Med 2007;19:1-8.

41. Fried AM, Woodring JH, Shier RW, Falace PB. Omphalocele in limb/body wall deficiency syndrome: atypical sonographic appearance. J Clin Ultrasound 1982;10:400-2.

42. Emanuel PG, Garcia GI, Angtuaco TL. Prenatal detection of anterior abdominal wall defects with US. Radiographics 1995;15:517-30.

43. Sohaey R, Woodward P, Zwiebel WJ. Fetal gastrointestinal anomalies. Semin Ultrasound CT MR 1996;17:51-65.

44. Servaes S, Victoria T, Lovrenski J, Epelman M. Contemporary pediatric gynecologic imaging. Semin Ultrasound CT MR 2010;31:116-40.

45. Chen CP. Prenatal sonographic diagnosis of limb-body wall complex with craniofacial defects. Ultrasound Obstet Gynecol 2003;22:101.

46. Colpaert C, Bogers J, Hertveldt K, Loquet P, Dumon J, Willems P. Limb-body wall complex: 4 new cases illustrating the importance of examining placenta and umbilical cord. Pathol Res Pract 2000;196:783-90. 